\title{
Diseño artesanal de tapabocas producto de la pandemia COVID-19. Una perspectiva Etnomatemática
}

\section{Handmade design of masks product of the COVID-19 pandemic. An \\ Ethnomathematical Perspective}

\author{
Nicanor Jaraba-Salcedo \\ Universidad del Atlántico, Barranquilla, Colombia \\ Rafael Martínez-Fonseca (D) \\ Universidad del Atlántico, Barranquilla, Colombia \\ Armando Aroca-Araújo \\ Universidad del Atlántico, Barranquilla, Colombia
}

Open Access:

ISSN: $0124-2121$

E-ISSN: $2665-2420$

\section{Resumen}

Objetivo: describir la geometría y procesos de medición desarrollados en el diseño y medición en un caso de confección artesanal de tapabocas que emergió en medio de la COVID-19. Método: La metodología empleó un enfoque cualitativo utilizando un diseño etnográfico con un análisis descriptivo, por medio de entrevistas semiestructuradas y observación participante. Se analizaron dos prácticas artesanales de modistas que laboran en talleres de confección ubicados en la ciudad de Barranquilla y Soledad, Atlántico, Colombia Resultados: Entre los resultados encontrados se describieron aspectos geométricos tales como la medición de longitudes en la tela, estimaciones de cantidades de piezas de tela a través de operaciones aritméticas, diseño de figuras, comparación de figuras a partir de las medidas de sus lados, construcción de figuras a partir de su área, medida de la superficie de figuras. Discusión y Conclusiones: En el artículo se discute a manera de comparación con respecto de otras investigaciones realizadas sobre las actividades de Diseñar y Medir y finalmente se concluye que se logró describir una geometría que se encuentra inmersa en la práctica de la confección de tapabocas mostrando un potencial aporte a la educación matemática para poblaciones escolares específicas, siendo este uno de los propósitos del Programa Etnomatemática.

Palabras clave: Etnomatemática, diseñar, sistema de medidas, práctica artesanal, COVID19.

\begin{abstract}
Objective: describe the geometry and measurement processes developed in the design and measurement in a case of handmade mask making that emerged in the midst of COVID-19. Method: through semi-structured interviews and participant observation, the methodology used a qualitative approach using an ethnographic design with a descriptive analysis. Two artisan practices of dressmakers working in tailoring workshops located in the city of Barranquilla and Soledad, Atlántico, Colombia were analyzed. Results: Among the results found, geometric aspects were described such as the measurement of lengths in the fabric, estimates of the quantities of pieces of fabric through arithmetic operations, design of figures, comparison of figures from the measurements of their sides, construction of figures from their area, measured from the surface of figures. Discussion and Conclusions: The article discusses by way of comparison with other investigations carried out on the Design and Measure activities and finally it is concluded that it was possible to describe a geometry that is immersed in the practice of making masks showing a potential contribution to mathematics education for specific school populations, this being one of the purposes of the Ethnomathematics Program.
\end{abstract}

Keywords: Ethnomathematics, design, measurement system, craft practice, COVID19.

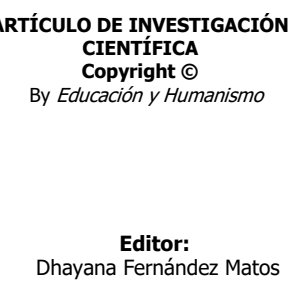

Correspondencia: armandoaroca@mail.uniatlan tico.edu.co

Recibido: $30-10-2020$ Aceptado: 25-05-2021 En línea desde: $14-10-2021$

Cómo citar este artículo (APA): Jaraba-Salcedo, N., Martínez-Fonseca, R. y Aroca-Araújo, A. (2021). Diseño artesanal de tapabocas producto de la pandemia COVID-19. Una perspectiva Etnomatemática. Educación y Humanismo, 23(41), 254-276. https://doi.org/10.17081/eduhum.23.41.4451 


\section{Introducción}

A continuación, se presentará una conceptualización sobre el Programa Etnomatemática, la actividad de Diseñar y Medir, un estado del arte sobre investigaciones afines sobre el objeto de estudio de esta investigación, la descripción del problema, su formulación y objetivo principal de la investigación.

\section{Una conceptualización sobre Etnomatemática}

La Etnomatemática es la matemática que se desarrolla y se practica en grupos culturales, tales como: comunidades urbanas y rurales, grupo de trabajadores, clases profesionales, sociedades indígenas, y todos aquellos grupos que poseen características comunes en su diario vivir, que siguen objetivos y tradiciones comunes, (D'Ambrosio, 2005).

Bishop (2005) plantea que las culturas generan matemática de la misma forma como generan su lenguaje y las costumbres propias del grupo, por lo que afirma que la matemática es un producto cultural, su desarrollo involucra actividades del medio, del entorno cultural del estudiante, Bishop considera que existen seis actividades "universales" involucradas en el desarrollo de pensamiento matemático como contar, localizar, medir, diseñar, jugar y explicar. Según los resultados de nuestra investigación se puede afirmar que hay mayor desarrollado de dos de estas actividades universales, diseñar y medir, en las prácticas artesanales de la confección de tapabocas que fueron estudiadas. Paralelo a esto Enríquez et al. (2012) afirman que la Etnomatemática brinda un acercamiento al objeto de estudio, pues por medio de esta, se emplea la diversidad de prácticas y saberes de un determinado grupo, dejando claro que enseñar matemáticas desde esta perspectiva, implica tener en cuenta la cultura de las personas.

Por su parte, Aroca (2013) plantea que las personas adquieren su propia cultura mediante el aprendizaje de las costumbres, creencias, lenguajes y técnicas de su grupo o comunidad, y de la misma manera su cultura matemática, a esto el autor le llama matemáticas culturales o Etnomatemática. De acuerdo con Gerdes (2014), la Etnomatemática hace referencia a la relación entre la antropología cultural, la Matemática y la Educación Matemática, manifestando que cada cultura posee su forma particular de desarrollar matemática.

Teniendo en cuenta lo planteado anteriormente sobre el Programa Etnomatemática, es pertinente afirmar que él busca el análisis y el estudio de la matemática que se encuentra inmersa en grupos o comunidades, que se dedican a prácticas culturales o sociales que día a día se enfrentan a situaciones de análisis y diversos procesos de cálculo para desarrollar un producto o artefacto determinado. Estos análisis por parte de los artesanos regularmente tienen su construcción en la experiencia, y también regularmente los mismos artesanos consideran que esos procesos de cálculos o análisis no son matemáticos. 
Es común, sobre todo en Brasil, que la palabra Etnomatemática se analice a partir de su etimología como el conjunto de modos, maneras, estilos, artes y técnicas (thica) que se utilizan para explicar, aprender, conocer, lidiar, enseñar (mathema) en ambientes naturales, sociales y culturales de una determinada cultura (etno), es decir, las ticas de mathema en una etno, (D'Ambrosio, 2014). Sin embargo, Aroca (2016) al respecto, plantea que esta definición etimológica planteada por D’Ambrosio, necesita de un análisis más profundo, en particular sobre las raíces de la palabra mathema y la relación de la palabra tics con technè, afirma que las múltiples interpretaciones que han hecho diferentes autores sobre estas dos raíces tics y mathema, se han alejado de su origen etimológico, a esto el autor lo denomina abuso etimológico y considera algunas implicaciones que esta definición de la Etnomatemática tendría en la educación matemática y en la investigación misma en el Programa Etnomatemática.

Por otra parte, Rosa et al (2016) platean que todas las culturas emplean y desarrollan formas, estilos y técnicas para llevar a cabo el trabajo del día a día, están en un constante aprendizaje, buscan diariamente respuestas y analizan constantemente el cómo y el porqué de las cosas. El enfoque Etnomatemático permite el aprendizaje de conceptos geométricos, por medio de artefactos o artesanías culturales, por lo que es importante reconocer el conocimiento matemático inmerso en la creación de artefactos culturales, donde se pone al descubierto el desarrollo de las actividades diarias de la cultura, (Rosa et al, 2017).

Por lo tanto, es conveniente afirmar que la naturaleza de las actividades artesanales, en nuestro caso la elaboración de tapabocas, incluye procesos diversos de cálculo, diseño y medición para obtener un producto específico.

De manera similar, D'Ambrosio (2018) plantea que la Etnomatemática es la matemática o construcciones abstractas de las regiones y civilizaciones de todo el mundo, civilizaciones integradas por artesanos, profesionales, grupos de personas que buscan objetivos comunes y considera que puede haber algunas similitudes matemáticas en diferentes civilizaciones, pero en esencia son distintas debido al permanente cambio.

De acuerdo con D'Ambrosio y Knijnik (2020) hacer Etnomatemática implica realizar trabajo de campo donde se utilizan técnicas etnográficas tales como la observación participante, diario de campo, grabación de audio y entrevistas, sin embargo; la Etnomatemática no solo implica Etnografía, pero si utiliza algunos de sus elementos, la Etnomatemática relaciona la investigación de campo y el trabajo pedagógico desarrollado en la escuela.

Por lo tanto, la matemática, específicamente la geometría que se encuentra inmersa en la práctica artesanal de elaboración de tapabocas responde a una perspectiva Etnomatemática, es una geometría que se da en el proceso mismo de la confección, una geometría contextualizada, una cultura específica dedicada a una actividad en común, que por motivos de la COVID-19 han cambiado las prendas que antes diseñaban, por la 
producción de tapabocas como comúnmente lo llaman en Colombia, pues su nombre técnico es mascarilla de bioseguridad. Esta expresión de tapabocas no es muy preciso debido a que no involucra la nariz.

Teniendo en cuenta lo planteado por Sánchez y Segura (2020), la economía, el sistema educativo y otros aspectos de la vida han sufrido transformaciones debido a la llegada de la pandemia COVID-19, generando estancamiento económico en los países. Una de las transformaciones más frecuentes que generó la pandemia COVID-19 en la vida de las personas, fue el uso diario del tapabocas, esto llevo a la comunidad de modistas a realizar cambios en sus productos, para dedicarse a la confección diaria de tapabocas, observando en este producto una forma viable de estabilizar su economía familiar durante este tiempo de pandemia.

Anteriormente las modistas se dedicaban a la confección de ropa deportiva y al diseño de vestidos para mujer, pero debido a la situación sanitaria actual que se vive a nivel mundial, la confección de tapabocas se convierte en una práctica artesanal emergente en el marco de la COVID-19, por lo que las modistas entrevistadas cambian varias formas de cortar, medir, diseñar, inferir, etc.

Según Fuentes (2010), debido al momento histórico-social y político que atravesaban las pymes textileras, su subsistencia y competitividad se encontraban amenazadas por el aumento desmesurado de precios de la materia prima; esto significaba para las modistas cambios drásticos en la administración de las telas utilizadas en sus talleres de confección, por lo que ellas se vieron en la difícil tarea de incluir procesos de optimación para el mayor aprovechamiento de la tela.

En consecuencia, a lo planteado anteriormente, es evidente el uso de la matemática por parte de estas modistas para enfrentar el problema de la optimización de la tela, incluyendo el análisis de situaciones reales de cálculos que seguramente las conducirán a un mejor aprovechamiento de la materia prima utilizada.

\section{Las actividades universales de Diseñar y Medir}

La Etnomatemática como una actividad universal se encuentra presente en los contextos socioculturales, Alan Bishop presenta seis actividades básicas para el desarrollo del pensamiento matemático, la actividad de contar, medir, localizar, diseñar, jugar y explicar. En esta investigación podemos resaltar las actividades de Diseñar y Medir como las actividades predominantes en la práctica de la confección de tapabocas como comúnmente se conocen en Colombia. Esto no quiere decir que otras actividades como Contar y Explicar se empleen.

A continuación, se relacionan algunas categorías que emergen sobre las concepciones de la actividad de diseñar. 


\section{Diseñar como actividad de la transformación de la materia:}

Según Bishop (2005) el diseño está relacionado con las transformaciones que se le realizan a la naturaleza u otros objetos para imponer una estructura particular deseada. Diseñar también implica la abstracción mental del objeto que se quiere obtener durante el proceso, las actividades de diseño involucran la tecnología, los artefactos y objetos manufacturados que las culturas producen para su vida y la medición es una de las actividades más importantes en el desarrollo de la matemática, esta actividad involucra diferentes acciones tales como comparar, ordenar y cuantificar.

\section{Diseñar como creatividad en el espacio:}

Para Alsina (2005) la actividad de Diseñar está intrínsecamente relacionada con la geometría, plantea que esta se relaciona con la creatividad en el espacio tridimensional, por lo que la geometría está presente en la creación de diseño, también afirma que el diseño busca soluciones geométricas para otorgar forma y medida a los objetos, los cuales serán diseñados para cumplir funciones determinadas a favor de la comunidad o cultura.

A continuación, se relacionan algunas categorías que emergen sobre las concepciones de la actividad de medir.

\section{Medir como actividad cotidiana, comercial y social:}

Zapata \& Cano (2008) afirman que la actividad de medir se puede apreciar desde distintos ángulos o aspectos sociales del hombre, desde las primeras civilizaciones hasta hoy, donde su uso es crucial para efectuar cualquier tipo de actividades comerciales y de la vida cotidiana.

\section{Medir como proceso derivado de un contexto en particular:}

Por su parte Gerdes (2013) plantea que de acuerdo con el contexto donde se encuentre una persona o una determinada cultura, la actividad de medir se manifiesta de maneras distintas, debido a que cada comunidad o cultura desarrolla su propia matemática y su sistema de medida. La actividad de medir está presente en muchos aspectos de la cotidianidad.

\section{Medir como actividad de comparación:}

De acuerdo con Rey \& Aroca (2011) la actividad de medir tiene como fundamento principal la comparación, la ordenación y la cuantificación de cualidades que poseen un valor, los autores aclaran que el proceso de medir se encuentra implícito en la tarea cotidianas dentro de las culturas de la humanidad en general.

Para Mengual et al (2017) los procesos que incluyen la actividad de medir, resultan complejos, debido que la medición encierra muchos conceptos fundamentales tales como la 
comparación, conservación de la idea de atributo, la conservación, la transitividad, la igualdad de particiones o la iteración de la unidad estandarizada.

De acuerdo con la modista Ana el diseño es inventar una nueva moda, por ejemplo; cuando se dispone a confeccionar una pantaloneta deportiva, comienza a inventarle nuevas cosas a la pantaloneta, desecha detalles viejos e impone los nuevos, de la misma manera ocurre con el diseño de los tapabocas, las modistas realizan transformaciones que son nuevas y desechan las viejas. Por su parte, la modista Fabiola plantea que diseñar es crear algún objeto nuevo, fabricar algo, por ejemplo; si se dispone a diseñar un nuevo vestido, afirma que debe crear moldes nuevos que ella misma los haga, inventar los moldes con creatividad.

Por lo tanto, teniendo en cuenta la práctica artesanal de la confección de tapabocas, entendemos por diseñar, a la transformación realizada a la materia prima, en este caso tela quirúrgica, mediante un proceso de abstracción mental del producto final (tapaboca), donde la geometría juega un papel importante y por ende los resultados son susceptibles de problematizarse en aula de clases de matemáticas. Sin embargo, esto último no se contempla en los objetivos de este artículo.

\section{Investigaciones afines}

A continuación, se presentan algunas investigaciones que se han hecho donde se analizaron las actividades de diseño, medición y confección. No fue posible encontrar una investigación que presente resultados sobre la confección de tapabocas.

Para Proenza \& Leyva (2008) el pensamiento geométrico es basado en el conocimiento de un modelo del espacio físico tridimensional y que también desarrolla tres capacidades, tales como: vista espacial, representación espacial e imaginación espacial. Romero, Juárez \& Navarro (2009), analizaron la geometría del Poncho Catamarqueño una prenda de vestir para invierno, presentaron una relación geométrica donde se pueda expresar métricamente la razón del porque la belleza y armonía de la prenda en sus medidas. Pérez (2013) afirma que el desarrollo del pensamiento geométrico involucra situaciones del entorno social, las cuales exigen estimaciones sobre formas, distancias y áreas, también plantea que las prácticas que incluyen geometría proporcionan creatividad, imaginación y el desarrollo de la capacidad deductiva.

Fuentes (2014) realizó un estudio de caso con un grupo de 8 modistas, donde el problema que aborda dicha investigación tiene que ver con el aumento significativo del precio de los materiales, por lo que las modistas se vieron obligadas a buscar estrategias para el máximo aprovechamiento de la materia prima. Los hallazgos encontrados por el autor fueron las estrategias, habilidades y pensamientos geométricos que utilizaron estas mujeres para aprovechar las telas al diseñar camisetas en un contexto laboral.

Bernabeu \& Llinares (2017) hablan sobre el reconocimiento de las figuras, plantean que 
lo perceptual influye de manera positiva en el reconocimiento de figuras, es decir, realizando comparaciones con objetos de la vida real, a este proceso el autor lo denomina aprehensiones perceptuales.

Morales \& Autor (2019) analizaron el diseño de las artesanías de Usiacurí, donde resaltan las diferentes configuraciones geométricas que hacen de la práctica del diseño una Etnomatemática, se describieron algunas figuras abstractas en dichas artesanías, donde la forma juega un papel muy importante en su diseño.

Se puede considerar que hay variedad de investigaciones que aportan al pensamiento geométrico a partir del diseño y la medición. La actividad Diseñar va ligada con aspectos geométricos, debido a la abstracción mental de formas y medidas que el diseñador debe tener en cuenta para su creación, así como su imaginación. Así la actividad Medir subyace, en este caso que se analizó, a la actividad Diseñar.

Por lo tanto, la Etnomatemática persigue esa relación que existe entre las prácticas desarrolladas en una cultura y la matemática que se desarrolla en una determinada cultura, en una práctica determinada. Así, lo anterior nos conduce a reflexionar lo siguiente: reconocer los diferentes sistemas de medición de las comunidades contribuyen a procesos de aceptación de las diferencias, de inclusión, del reconocimiento de los demás. Esto motiva a reconocer las diferentes formas de medir en contextos locales, por ejemplo, en una práctica artesanal, se trata del reconocimiento de otras formas de pensar matemáticamente.

\section{Problema y formulación de la investigación y objetivo principal}

El problema de esta investigación consistió en la necesidad de distinguir otras formas no reconocidas de pensamiento matemático, más específicamente geométrico, que se desarrollan en las actividades de diseño y medición inmersas en la práctica artesanal de la confección de tapabocas que emergió en medio de la pandemia de la COVID-19¹.

El objetivo general fue describir la geometría desarrollada en los procesos de diseño y medición en un caso de confección artesanal de tapabocas que emergió en medio de la COVID-19, de donde surgió una pregunta: ¿qué aspectos geométricos se pueden describir en los procesos de diseño y medición inmersos en la práctica artesanal de la confección artesanal de tapabocas?

\section{Método}

Presentaremos a continuación algunas características básicas que se desarrollaron en la metodología de esta investigación, tales como: el contexto y participantes, el tipo de

\footnotetext{
${ }^{1}$ Según la Organización Mundial de la Salud, OMS (2020), el uso del tapabocas puede ser útil para prevenir la transmisión de virus como el COVID-19, donde se recomienda la producción en masa de tapabocas caseros y fabricados en talleres de confección caseros.
} 
investigación que se realizó, las diferentes técnicas e instrumentos de recolección de información e instrumentos de observación.

\section{Tipo de investigación}

Se plantea esta investigación con un enfoque cualitativo y un alcance descriptivo, a partir de un diseño etnográfico. Este marco metodológico se asume a partir de Vasilachis (2006) y Hernández (2014).

\section{Método de investigación}

El método de esta investigación fue asumido a partir de Hernández (2014) el cual plantea que uno de los diseños de una investigación cualitativa es el diseño etnográfico, este es de carácter holístico ya que inicialmente busca una perspectiva general y después se va enfocando en lo particular, es decir, en aquellos elementos que tienen mayor relevancia para interpretar al grupo, comunidad o cultura. Así, se parte de una idea general con respecto a las prácticas realizadas por las modistas a nivel nacional, diferentes tipos de confecciones y prendas elaboradas por ellas para luego centra el análisis en elementos específicos, de tipo matemático, de la confección de tapabocas.

De manera similar, Vasilachis (2006), afirma que la investigación cualitativa se ocupa de la vida de la persona, de historias, de comportamientos, del funcionamiento organizacional, de los movimientos sociales, basada en la comunicación en la recolección de historias, narrativas y descripciones de las experiencias de otro.

Siguiendo lo planteado por Vasilachis, esta investigación se basó en la comunicación activa con las modistas, en la recolección de ideas de las modistas, de sus historias, sus narrativas y las descripciones de sus experiencias.

\section{Técnicas de recolección de información}

Las principales técnicas de recolección de información se basaron en entrevistas semiestructuradas, transcripciones de registros audiovisuales empleando los signos Val.Es.Co, notas de campo, registros audiovisuales, fotografías y la observación participante, donde los autores de este artículo también son artesanos, participan del proceso de elaboración de los tapabocas.

\section{Instrumentos de recolección de información}

El instrumento de recolección de información utilizado en esta investigación fue un cuestionario construido con 90 preguntas, basado en el proceso artesanal de la elaboración de tapabocas, el cual fue aplicado a las modistas mediante entrevistas semi estructurada. Se suma a este instrumento el diario de campo. 


\section{Contexto, población y muestra}

La práctica artesanal en la cual se basó esta investigación fue la confección de tapabocas. La práctica artesanal analizada emergió en medio de la pandemia producida por la COVID19. Esta emergencia ha implicado la reinvención de varias empresas nacionales que están produciendo ahora suplementos médicos y productos sanitarios para abastecer clínicas y hospitales colombianos, por ejemplo, hay empresas locales que producen más de 50.000 tapabocas diarios (Fashion Revolutions Colombia, 2020).

La población son las modistas de Barranquilla y la muestra fue dos modistas de las ciudades de Barranquilla y Soledad, Atlántico, Colombia, las cuales en estos momentos se dedican a la elaboración de tapabocas. Los datos generales de las modistas son:

\section{Tabla 1}

Datos Generales de las Modistas

\begin{tabular}{cccc}
\hline Modista & Nivel de Escolaridad & Edad & Experiencia Laboral \\
\hline Ana & Primaria & 56 años & 25 años aprox. \\
Fabiola & Bachiller & 50 años & 20 años aprox. \\
\hline
\end{tabular}

Fuente: Elaboración propia (2020)

\section{Resultados}

El proceso de confección de tapabocas se realiza en 6 fases: Fase 1. Tendido, trazado y corte de la tela. Fase 2. Corte de elásticos. Fase 3. Cerrado de bordes. Fase 4. Realización de pliegues o dobles. Fase 5. Postura del bies y elásticos. Fase 6. Empacado y venta.

\section{Fase 1: Tendido, trazado y corte de la tela}

En esta fase la modista Ana primero desenrolla la tela con ayuda de otra persona, se dobla o se pliega la tela de tal forma que los extremos queden parejos, luego en la mesa de corte se colocan las capas de tela ya doblada para su tendido, se colocan 25 capas de tela quirúrgica en la mesa, luego con la ayuda de una regla de aluminio, bolígrafo y cinta métrica, comienza a realizar el trazo del tapaboca en forma de cuadrados de $(18 \mathrm{~cm} \times 18 \mathrm{~cm})$, posteriormente con ayuda de la cortadora se dispone a realizar el corte por la marca del trazo realizado, ver figura 1 . 
Figura 1.

Tendido de la tela, trazo y corte de los tapabocas

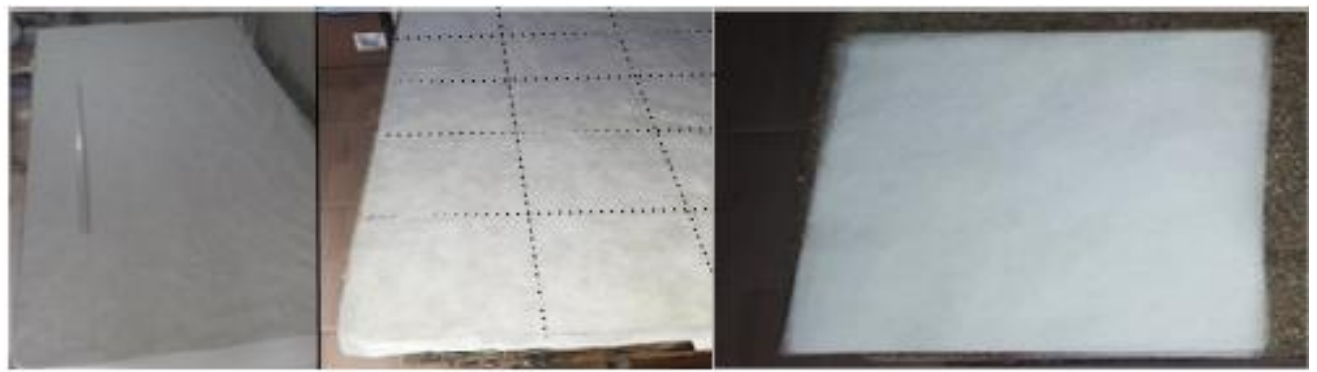

Fuente: Fotografía de los autores, trabajo de campo (2020)

A continuación, se muestra un bosquejo del trazado de formas cuadradas sobre el tendido de la tela con las medidas iniciales del tapaboca, se muestra el número de tapabocas resultantes de un tendido de tela y el número de capas de tela una sobre otra colocadas por la modista, ver figura 2.

Figura 2.

Bosquejo del trazado sobre el tendido de la tela

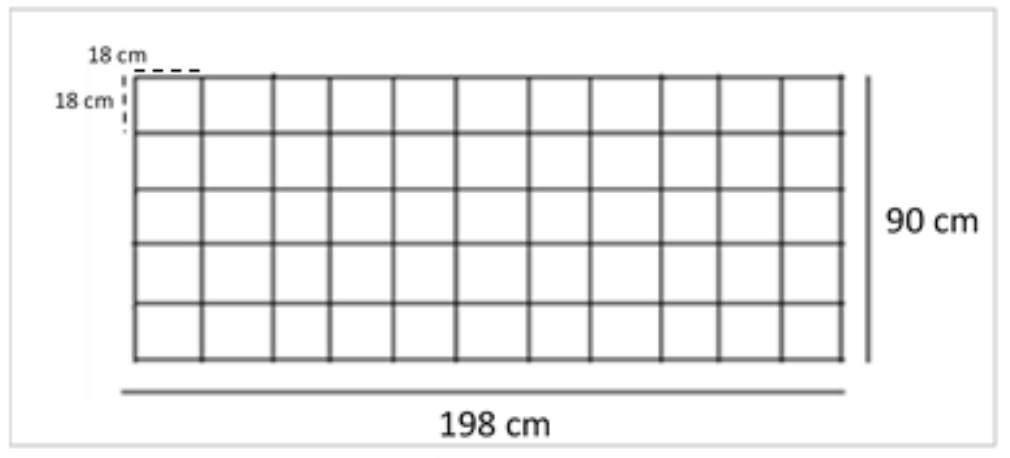

№ de capas $=25$

Fuente: Elaboración propia (2020)

En esta fase la modista ya sabe cuántos tapabocas resultan del corte a realizar, debido a que ella previamente realiza algunos cálculos de área sobre la tela tendida, así lo expresa la modista:

"...o sea de aquí me salen: de aquí me salen/// 55 porque me salen así 11// (se refiere a lo largo del tendido de la tela) por 5 (se refiere al ancho de la tela tendida) que son 55 / ese es un cuadrado de $18 \times 18$ / entonces de estas capas me salen ...5 / 55 piezas así por 25 capas que puse 5 por 525 y van 27-10-12/// me salen 1.475 mascarillas en este tendido/ porque va doble: esta es mascarilla doble." (Ana, 
comunicación personal, 1 de mayo de 2020)².

Como podemos observar, la señora Ana tiene en cuenta algunos aspectos geométricos para realizar el trazado sobre la tela tendida.

A continuación, se describen algunos aspectos geométricos que se desarrollan en esta primera fase:

Tabla 2

Aspectos Geométricos Descritos en la Primera Fase de la Confección

Descripción Datos Numéricos

Dimensiones del tapaboca

Superficie inicial del tapaboca

Dimensiones del rollo de tela

Dimensiones de la tela tendida

Cantidad de capas de tela plegada

Cantidad de piezas resultantes (largo)

Cantidad de piezas resultantes (ancho)
Largo: $18 \mathrm{~cm}$ - ancho: $18 \mathrm{~cm}$

$324 \mathrm{~cm}^{2}$

Largo: $512 \mathrm{~m}$ - ancho: $1,80 \mathrm{~m}$

Ancho: $90 \mathrm{~cm}$ - largo: $198 \mathrm{~cm}$

25 capas

11 piezas o cuadros

5 piezas o cuadros

Fuente: Elaboración propia (2020)

Mientras tanto la modista Fabiola, desenrolla y pliega la tela de 1,60 cm de ancho en dos partes iguales, por lo que le resultan capas de $80 \mathrm{~cm}$, posteriormente nuevamente realiza otro pliegue de la tela y finalmente le resultan capas de $40 \mathrm{~cm}$ de ancho, debido a que la mesa que utiliza no es muy grande y de esta manera se le facilita el corte. Para el trazado sobre la tela utiliza un molde rectangular de cartón de $(16 \mathrm{~cm} \times 20 \mathrm{~cm})$, una regla tipo T, lápiz blanco y cinta métrica, luego con ayuda de la tijera realiza el corte sobre el trazo marcado inicialmente, así lo expresa la modista:

"...Los bordes de la tela tienen que quedar parejitos por lado y lado/ doblada en dos uno sesenta de ancho/ nos daría una capa de 80 centímetros.../ la cual la volvemos a doblar en dos/ esa es la técnica que yo utilizo debido a que mi taller no es muy grande/ ni tengo mesas amplias y así se me facilita el corte/ En este momento hemos doblado la tela en 4 partes prácticamente iguales/ lo que le volví a decir/ el borde de la tela debe ir parejo para que el cortado pueda salir derechito/ ya la tela emparejada en cuatro nos daría capas de $40 \mathrm{~cm} /$ Bueno, salen 8 tracitos de tela me

\footnotetext{
${ }^{2}$ Los signos Val.Es.Co empleados para la transcripción de la anterior conversación con la señora modista fueron los siguientes : cambio de voz, /// pausa de un segundo o más, // pausa entre medio segundo y un segundo, / pausa corta, inferior al medio segundo, - reinicios y auto interrupciones sin pausa, $\ldots$ pausa pensativa con sonido de un segundo o más.
} 
darían 4 tapabocas porque van doble faz, doble filtro/ ¿cómo lo emparejo?/ ya lo tengo aquí entonces/ lo abro aquí/ le busco el pegue acá y sin necesidad de estar midiendo con el metro lo doblo en dos/ lo doblamos en dos y tenemos/ 8 papelitos/ 8 trocitos de tela/ que vienen a ser los dos un tapaboca rectangular que es la que estamos elaborando." (Fabiola, comunicación personal, 12 de mayo de 2020). Ver figura 3.

\section{Figura 3.}

Tendido de la tela plegada en partes iguales, trazo y corte de los tapabocas

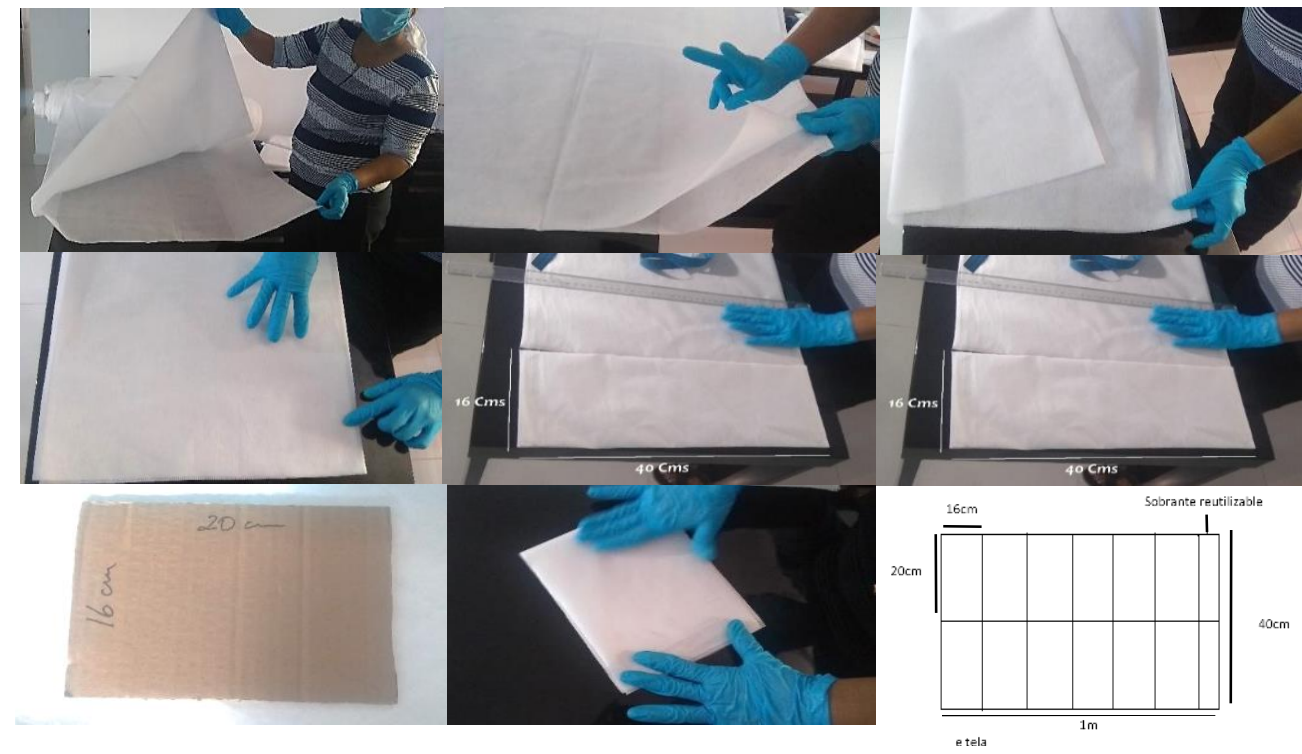

Fuente: Fotografía de los autores, trabajo de campo (2020)

La señora Fabiola tiene en cuenta algunos aspectos geométricos, optimizando así el máximo aprovechamiento de la tela. A continuación, se describen algunos aspectos geométricos que se desarrollan en esta primera fase:

Tabla 3

Aspectos Geométricos Descritos en la Primera Fase de la Confección (Modista 2)

\begin{tabular}{ll}
\hline \multicolumn{1}{c}{ Descripción } & \multicolumn{1}{c}{ Datos numéricos } \\
\hline Dimensiones iniciales del tapaboca & Largo: $20 \mathrm{~cm}-$ ancho: $16 \mathrm{~cm}$ \\
Superficie inicial de la mascarilla & $320 \mathrm{~cm}^{2}$ \\
Dimensiones del rollo de tela & Largo: $300 \mathrm{~m}-$ ancho: $160 \mathrm{~cm}$ \\
Cantidad de capas de tela plegada & 4 capas plegadas sobre la mesa en forma de \\
Cantidad de piezas que resultan & rectángulos de $40 \mathrm{~cm} \times 16 \mathrm{~cm}$ \\
Cantidad de mascarillas que resultan de un & 8 piezas \\
corte rectangular & 4 mascarillas
\end{tabular}


Cantidad de mascarillas que resultan de un 1 metro de tela

Cantidad de mascarillas que resultan del rollo de tela de 300 metros $\frac{100 \mathrm{~cm}}{16 \mathrm{~cm}}=6$ mascarillas aprox.

$6 \times 4=24$ mascarillas que resultan de 1 metro de tela. Sobrante $4 \mathrm{~cm}$

$24 \times 300=7.200$ Mascarillas

Fuente: Elaboración propia (2020)

\section{Discusión}

\section{El potencial aporte a la educación geométrica}

La enseñanza de la geometría también implica relacionar el contexto sociocultural con la temática desarrollada en el aula de clases. No es fácil encontrar contextos del ser humano donde la geometría no aparezca de forma directa o indirecta, por tanto, se hace necesario relacionar la geometría con otras áreas del saber (Báez e Iglesias, 2007). De acuerdo con Verner et al (2019), los estudiantes prefieren un aprendizaje contextualizado. Enseñar matemática implica entonces la conexión del estudiante con las prácticas del mundo real y las ideas matemáticas entre lo visual-intuitivo y lo racional-lógico, también plantean que entre los principales aportes a la educación geométrica se consideran varios factores influyentes tales como, el contexto, efecto, cultura y etnia, por lo que es necesario construir una cultura escolar.

La importancia de esta investigación se relaciona con el potencial aporte a la educación geométrica que esta brinda, donde los hallazgos que se describieron en cada una de las fases de la práctica artesanal de la confección de tapabocas pueden ser comparados por medio del diseño de actividades académicas donde estas pueden ser problematizadas en aulas de clases de geometría.

\section{Fase 2: Corte de elásticos}

Los elásticos son parte del tapaboca, su presentación es en rollos de 300 metros, debido a la situación pandémica los materiales se han escaseado por lo que a veces el elástico lo venden por metraje o sea por pedazos. La función de las modistas en esta fase es cortar los elásticos que vienen en rollos a la medida necesaria, para esto crearon una marca en la superficie de corte o mesa con la longitud del elástico, teniendo ya la medida proceden con el corte, para este procedimiento utilizan como herramienta la tijera y la cinta métrica para la marca inicial, ver Figura 4. 
Figura 4.

Presentación y corte de elásticos

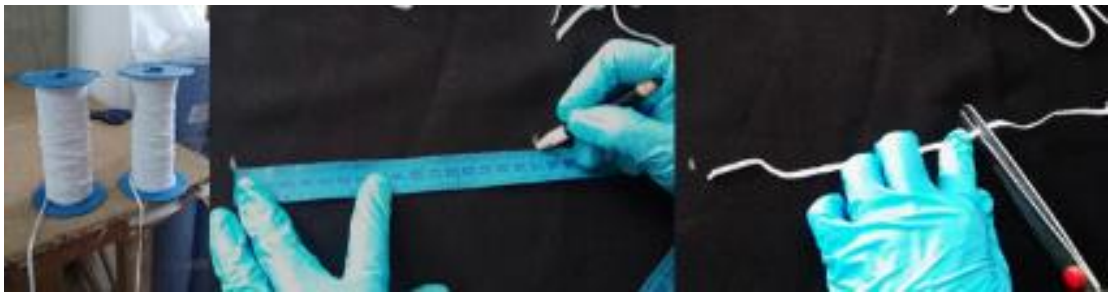

Fuente: Fotografía de los autores, trabajo de campo (2020)

El siguiente pasaje nos suministra información del proceso de corte de los elásticos:

I. ¿De qué forma vienen los elásticos?

P. Vienen en forma de rollos/ de 100 de 200 y 300 metros

I. ¿Qué largo deben tener los elásticos de Los tapabocas?

P. de 16 a $17 \mathrm{~cm}$

I. ¿Cómo mide usted este largo?

P. bueno/ yo tengo una marquita en la mesa/ y voy cortando/ mido los $16 \mathrm{~cm} /$ tengo 16 y $18 \mathrm{~cm}$ la marca.

I. ¿Cómo realiza el corte de los elásticos?

P. bueno yo pongo los rollos de elásticos / y voy cortando según la medida del tapaboca, voy cortando de 5 en 5 depende la cantidad de rollos que tenga. (Ana, comunicación personal, 3 de mayo de 2020).

Se puede apreciar en la anterior conversación con la modista, el uso de la actividad de medir en la fase de corte de los elásticos. Durante este proceso de medición que la modista realiza en esta fase, se pudo evidenciar comparaciones y el uso de partes del cuerpo para obtener la medición, es decir, la modista en ocasiones precisa estos cálculos de medición de forma empírica, los cuales son importantes para un análisis etnomatemático y que podrían ser objeto de estudio en el contexto escolar, en aula de matemáticas.

Los resultados encontrados en esta investigación, es decir, aspectos geométricos inmersos en la confección de tapabocas tales como, medición de longitudes en la tela y elásticos, estimaciones de cantidades de piezas de tela a través de operaciones aritméticas, construcciones de figuras sobre la tela, pueden ser utilizados como principal insumo para la enseñanza paralela y comparativa de las matemáticas como propone Autor (2018). 


\section{Fase 3: Preparación de máquinas de coser y Cerrado de los bordes}

Una vez se tenga preparada y cortada las piezas de tela del tapaboca en su forma geométrica, se procede a cerrar la parte lateral superior, es decir; se procede con el cierre de dos de los cuatro lados de la pieza de tela rectangular o cuadrada según sea el caso o referencia, por parte de la modista Fabiola, utiliza una máquina fileteadora para realizar esta operación de cerrado y la modista Ana utiliza una máquina plana de costura recta, el cerrado de bordes del tapaboca sirve para sujetar las dos piezas de telas rectangulares o cuadradas que inicialmente fueron cortadas, ver Figura 5.

Figura 5.

Cerrado de bordes y máquinas de coser
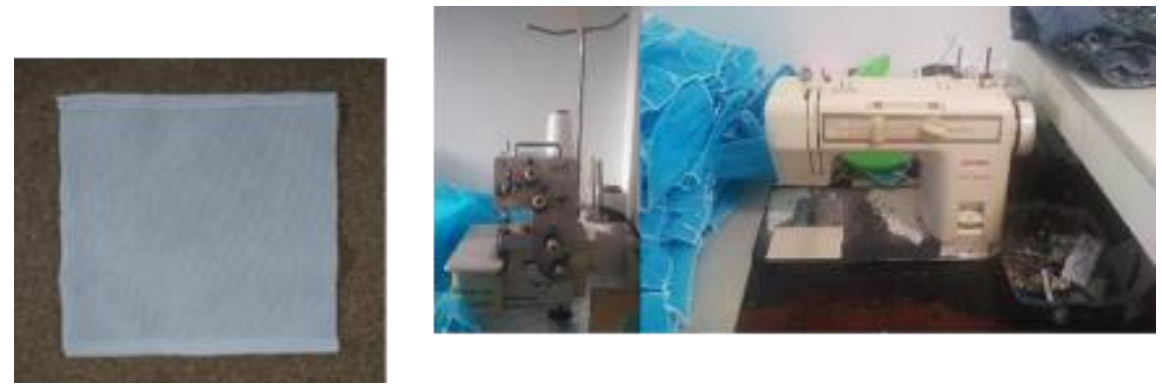

Fuente: Fotografía de los autores, trabajo de campo (2020)

\section{Fase 4: Realización de pliegues o dobles}

Una vez obtenida la pieza ya cerrada, se procede a realizar los pliegues del tapaboca, este proceso consiste en realizar dos o tres dobles sobre la tela según sea la referencia, aproximadamente de $1,5 \mathrm{~cm}$ de longitud, la función de estos pliegues es abrir y cerrar la mascarilla de acuerdo a su debido uso, estos pliegues son realizados a partir de un molde de cartón en forma de acordeón, para fijar los pliegues en la tela las modistas utilizan la máquina plana, debido a que solo es fijar y no pulir bordes, ver Figura 6 .

Figura 6. Pliegues realizados en el tapaboca

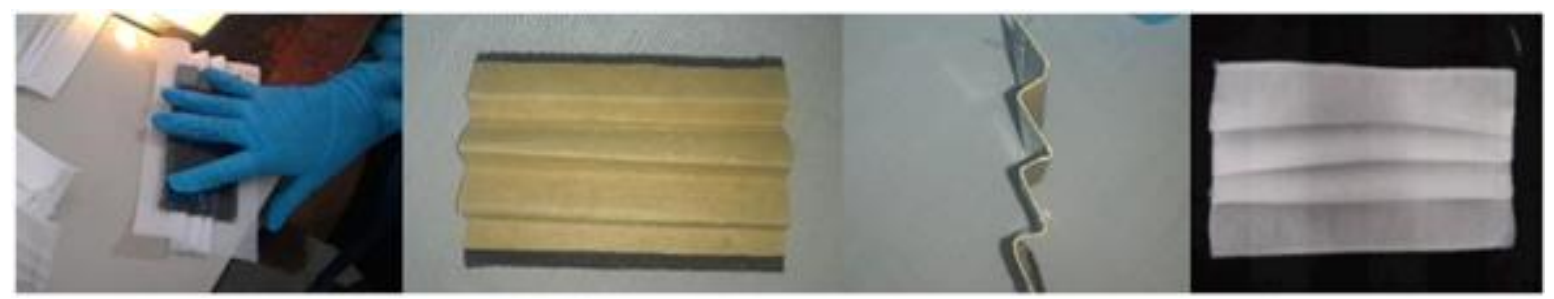

Fuente: Fotografía de los autores, trabajo de campo (2020)

\section{Fase 5: postura del bies y elástico}

El bies es un pedazo de tela muy delgada de $3 \mathrm{~cm}$ de ancho, el cual se utiliza para pegar 
el elástico en el tapaboca. En este proceso se observa que las señoras modista pueden hacerlo de dos formas, una de ellas es con el uso de un bies y la otra únicamente fileteando los laterales del tapaboca. El pegado del elástico se realiza utilizando un bies, uno de sus ayudantes en el taller nos expresa que:

"...Los elásticos los ubicamos al borde / porque es lo más conveniente, recuerda que llevamos los pliegues en el medio/ colocamos los elásticos al borde" (trabajador del taller, comunicación personal, 6 de mayo de 2020).

El pegado del elástico está vinculado con la forma en la que se usan los tapabocas, relacionado con la morfología de la cara humana, la señora Fabiola nos expresa lo siguiente:

"...la mascarilla lleva los elásticos en los lados laterales, derecho- izquierdo, porque al colocar la mascarilla así (en el rostro de la persona), estos inclinan hacia el odio, hacia la oreja derecha, hacia la oreja izquierda, entonces ese es el puesto de los elásticos." (Fabiola, comunicación personal, 25 de mayo de 2020).

Es usual que las señoras modistas realizan mediciones con el cuerpo de los clientes cuando normalmente se realiza la confección de prendas, en el caso de los tapabocas las modistas presentan el diseño bajo los patrones de forma en el rostro, como se muestra en la figura 7.

\section{Figura 7.}

Tapaboca terminado y patrones vinculados con el diseño

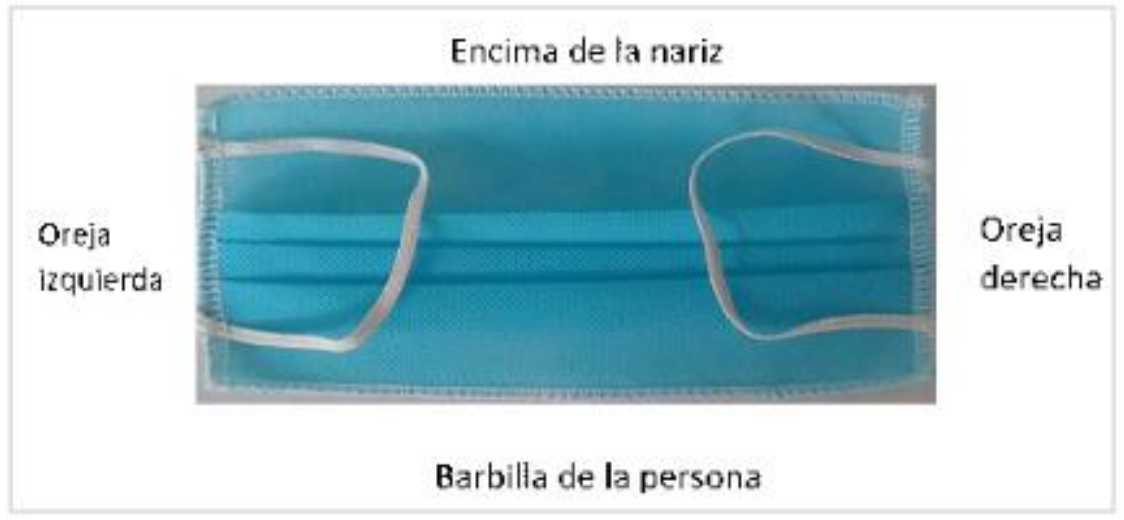

Fuente: Fotografía de los autores, trabajo de campo (2020)

\section{Fase 6: Empacado y venta de los tapabocas}

La señora Ana nos expresa:

"...se limpian se quitan los hilitos... se empaca / esta es una bolsita de 5x8 / las cajas que yo vento trae veinticinco, hay unas que son de cincuenta/ la caja vale 40 salen 
a 80 pesos cada una/ ya este se empaca en la bolsita/se sella y luego va al empacado". (Ana, comunicación personal, 10 de junio de 2020).

El producido final del taller se obtiene en cajas o paquetes con un conjunto de tapabocas determinado según el cliente, como se muestra en la figura 8.

Figura 8.

Producto terminado empacado en el taller

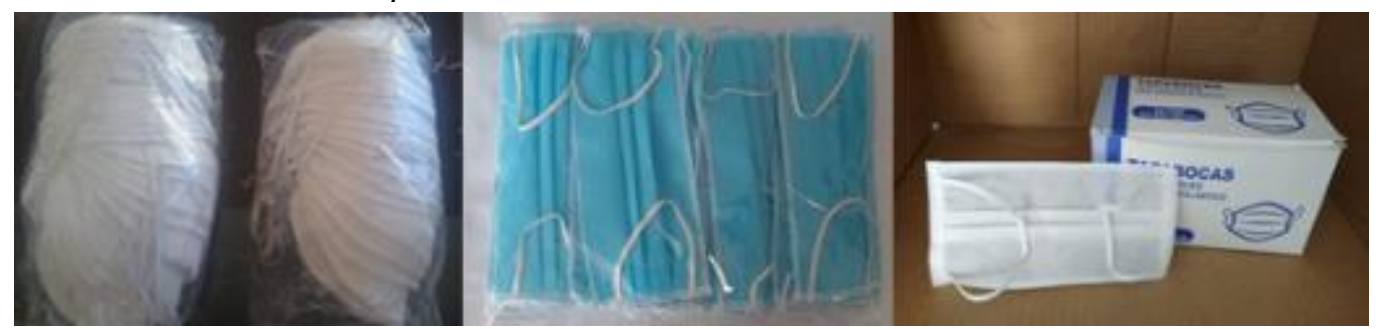

Fuente: Fotografía de los autores, trabajo de campo (2020)

Las señoras modistas comunican que estos paquetes son distribuidos a empresas solicitantes del producto y a vendedores individuales, los paquetes se transportan a su destino por domicilio y entregas directas, algunas empresas envían a su mensajero a recoger el paquete que previamente fue sellado muy bien para su transporte y en otras ocasiones el paquete es enviado a su lugar destino por mensajeros particulares y empresas oficiales dedicadas al transporte de paquetes.

Podemos observar en la siguiente figura la geometría empleada en otros diseños elaborados en el taller, ver figura 9.

Figura 9.

Otros diseños elaborados en el taller

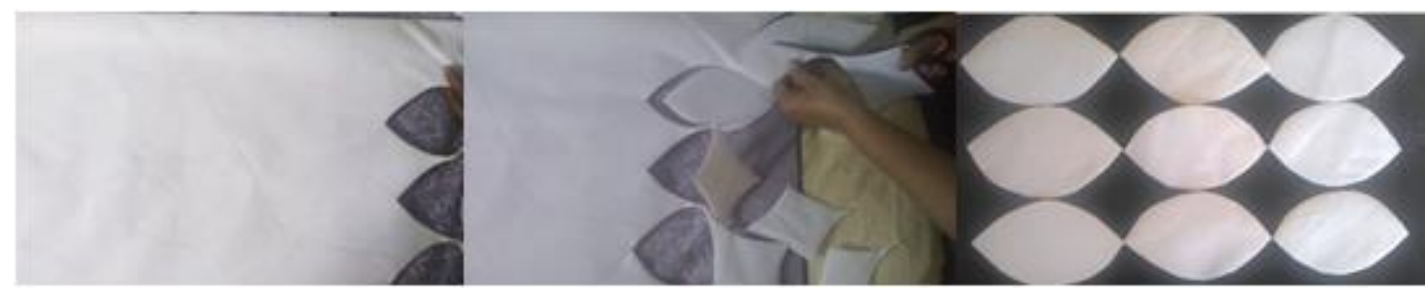

Fuente: Fotografía de los autores, trabajo de campo (2020)

De acuerdo con los resultados obtenidos, se pueden describir algunos aspectos geométricos que se desarrollan durante las fases de la práctica artesanal de la confección del tapaboca, aspectos tales como; medición de longitudes en la tela, estimaciones de cantidades de piezas de tela a través de operaciones aritméticas, construcciones de figuras, comparaciones de figuras a partir de la medida de sus lados, propiedades de figuras involucradas en el proceso de medición, identificación, construcción y comparación de figuras a partir de su área, medida de la superficie de figuras. 
Finalmente se discute de manera general a partir de los fundamentos teóricos de esta investigación a manera de comparación sobre las actividades de diseño y medición.

La realización de esta práctica de la confección requiere algunos procesos mentales tales como imaginar, transformar, crear. Las modistas imaginan, transforman y crean lo cual obedece a lo planteado por Bishop (1999), el diseño está relacionado con las transformaciones que se le realizan a la materia para imponer una estructura particular deseada, diseñar también implica la abstracción mental del objeto que se quiere obtener durante el proceso, las actividades de diseño involucran la tecnología, los artefactos y objetos manufacturados que las culturas producen para su vida.

De esta manera en las en la elaboración de tapabocas se diseña a partir de formas geométricas como se registró en los resultados. Bishop (1999) afirma:

El diseño de objetos ofrece la posibilidad de imaginar formas, Figuras y pautas en el entorno. Naturalmente, eso no significa que las formas, las Figuras y las pautas no se den en el entorno natural, sino que cuando las formas se trazan, realizan y diseñan las formas mismas se convierten en el centro de atención. (p.61)

Siguiendo con este corte de discusión se realizará una comparación con otras investigaciones sobre el diseño y la medición. Ampliaremos un poco más el contexto de algunas prácticas que utilizan la comparación, ordenación y cuantificación, las cuales obedecen a la actividad de Medir planteada por Alan Bishop, para esto presentaremos algunas investigaciones tales como Rey \& Autor (2011) donde plantean que existen puntos o lugares de referencias los cuales son métodos para estimar una medida determinada, estos son puntos o lugares de referencias que se utilizan para estimar la posible medida de una longitud, podemos observar que existe una acción comparativa, la cual dicha acción es utilizada por las modistas para realizar la fase de corte de los elásticos durante la confección de tapabocas, ellas utilizan puntos o lugares de referencia para comparar la longitud del elástico a cortar, se evidencia la actividad universal de la medición.

Por su parte Fuentes (2012), analizó propiedades geométricas involucradas en el diseño de las artesanías del municipio de Guacamayas, Boyacá, Colombia. Dentro de sus hallazgos, identifico actividades universales en las artesanías, actividades tales como Diseñar y Medir, donde los aspectos geométricos juegan un papel muy importante, afirma que algunos artesanos manejan representaciones abstractas y no físicas de los diseños a construir. De manera similar a estas conclusiones del autor, en esta investigación se hallaron algunos aspectos geométricos inmersos a las actividades de diseño y medición de la confección de tapabocas, como se pueden apreciar en los resultados.

Marmolejo \& Vega (2012), plantean que el aprendizaje de la geometría se da a través de la coordinación de tres actividades tales como: la visualización, razonamiento y construcción. Haciendo un paralelo, la visualización, el razonamiento y la construcción se da en la práctica 
artesanal de la confección de tapabocas.

Fuentes (2014), en su investigación con modistas de la ciudad de Bogotá, Colombia plantea que estas trabajan con un cuadrado de tela de $2 \mathrm{~m} \times 2 \mathrm{~m}$ y utilizaban el centro de este cuadrado para reflejar los moldes de igual forma, repitiendo esta técnica continuamente, por lo que el autor plantea que inicialmente hay ausencia de pensamiento matemático en esta práctica repetitiva, partiendo de esta situación el autor consideró proponer situaciones donde las modistas tuvieran que crear, idear respuestas a situaciones problemas de su contexto y donde tuvieran que pensar en soluciones alternas. De esta forma si había presencia de pensamiento matemático.

haciendo un paralelo con lo que plantea Fuentes en su investigación, podemos decir que en las prácticas realizadas por las modistas, si existe pensamiento matemático, aunque en su proceso de confección de tapabocas, existan algunos procesos de repetición constante, también hay procedimientos que implican creatividad, análisis, creación, comparación, ordenación, cuantificación y diseño, las cuales algunas de estas características obedecen a las actividades universales planteadas por el teórico Alan Bishop, por lo que se concluye presencia de pensamiento matemático en sus prácticas diarias de la confesión, es decir; matemática inmersa en sus prácticas.

García \& Bernandino (2019) notaron el uso de las medidas no estandarizadas como la cuarta para medir las dimensiones de güilile, la cual es una de las principales herramientas de trabajo de los hombres en la comunidad ñuu savi, las modistas también utilizan partes de su cuerpo para hacer comparaciones, para cuantificar y ordenar, lo cual obedece a la actividad de Medir planteada por Alan Bishop.

En esta discusión se pudo apreciar las similitudes y diferencias que existen entre otras investigaciones y el presente artículo, cabe resaltar que la descripción de los hallazgos encontrados en los resultados, presentan un potencial aporte a la educación matemática más específicamente geométrica. Las habilidades de pensamiento geométrico dependen de la abstracción mental y espacial, que el sujeto pueda realizar de un objeto.

\section{Conclusiones}

En síntesis, en esta investigación se describió una geometría naturalmente inmersa en la práctica artesanal de la confección de tapabocas, relacionada con las actividades de diseño y medición desarrolladas por las modistas.

A continuación, se describen los principales hallazgos geométricos encontrados en la práctica: 
1. Medición de longitudes en la tela

2. Construcción de figuras sobre la tela

3. Estimaciones de cantidades de piezas de tela a través de operaciones aritméticas

4. Estimaciones de cantidades de piezas de tela a través de operaciones aritméticas

5. Comparación de figuras a partir de las medidas de sus lados

6. Propiedades de figuras geométricas involucradas en el proceso de medición

7. Identificación, construcción y comparación de figuras geométricas a partir de su área

8. medición de la superficie de figuras sobre la tela

Además, las tomas de decisiones en el momento del proceso de elaboración de tapabocas están estrechamente relacionadas con situaciones problemas que viven día a día las modistas, de esto se puede concluir que las decisiones que hacen parte de la actividad diaria de las artesanas y que a larga hacen parte de un sistema de situaciones inesperadas que comúnmente implican pensamiento matemático. Estos procesos o acciones también podrían ser analizados en la enseñanza/aprendizaje en aulas de clases de matemáticas.

También se destacan las adaptaciones económicas y habilidades numéricas para el cálculo de costos de los materiales, viabilidad y ganancia por parte de las modistas. A partir de esta investigación pueden surgir otros posibles estudios con modistas a nivel nacional y local. Los resultados de esta investigación se asumen como un material potencial para el desarrollo de actividades significativas en el aprendizaje de la geometría, específicamente el cálculo de superficies pues puede permitir un diseño de situaciones problemáticas, contextualizadas, que contribuyan al proceso de enseñanza-aprendizaje en el aula de clases de matemáticas. Justamente, la segunda y final fase de la investigación que los autores están desarrollando implica la problematización de los resultados de la fase etnográfica en aulas de clases de matemáticas, partiendo de la enseñanza paralela y comparativa como identificación de a postura didáctica del Programa Etnomatemática propuesta por Aroca (2018).

\section{Referencias}

Alsina, C. (2015). Los secretos geométricos en diseño y arquitectura [Presentación]. Sociedad, Ciencia, Tecnología y Matemáticas. Universidad Politécnica de Cataluña, Barcelona, España.

Aroca-Araujo, A. A. (2013). Los Escenarios de Exploración en el Programa de Investigación en Etnomatemática. Educación Matemática, 25(1), 111-131. https://bit.ly/3eIY3T4

Aroca-Araujo, A. A. (2016). La definición etimológica de Etnomatemática e implicaciones en Educación Matemática. Educación Matemática, 28(2), 175-195. 


\section{https://doi.org/10.24844/EM2802.07}

Aroca, A. (2018). Enseñanza paralela y comparativa. La postura didáctica del programa Etnomatemática. En Valbuena, S.; Vargas, L.; Berrío, J. (Eds.), Encuentro de Investigación en Educación Matemática (pp. 475-481). Universidad del Atlántico.

Baez, R. e Iglesias, M. (2007). Principios didácticos a seguir en el proceso de enseñanza y aprendizaje de la geometría en la upel "el macaro". Enseñanza de la Matemática, 12(16), 67-68. http://funes.uniandes.edu.co/14702/1/Baez2007Principios.pdf

Bernabeu M. y Llinares S. (2017). Compresión de las figuras Geométricas en niños de 6 a 9 años. Educación Matemática, 29(2), 9-35. http://dx.doi.org/10.24844/em2902.01

Bishop, A. (2005). Aproximación sociocultural a la educación matemática. Editorial Universidad del Valle.

Bishop. A. (1999). Enculturación Matemática, la educación matemática desde una perspectiva cultural. Paidós Ibérica.

Comisión Económica para América Latina y el Caribe. (2020, 21 de abril). Pandemia del COVID-19 llevará a la mayor contracción de la actividad económica en la historia de la región: caerá -5,3\% en 2020 [comunicado de prensa]. https://www.cepal.org

D'Ambrosio, U. y Knijnik G. (2020). Ethnomathematics. In S. Lerman (Ed.), Encyclopedia of Mathematics Education (pp. 283-287). Springer.

D'Ambrosio, U. (2005). Etnomatemática. Elo entre as tradições e a modernidades. Coleção Tendências em Educação Matemática. Autêntica Editora.

D'Ambrosio, U. (2014). Las bases conceptuales del Programa de Etnomatemática. Revista Latinoamericana de Etnomatemática, 7(2), 100-107.

D'Ambrosio, U. (2018). The program Ethnomathematics: Cognitive, anthropological, historic and socio-cultural bases. PNA, 12(4), 229-247. http://hdl.handle.net/10481/53007

Enríquez, W., Millán, B., y Aroca-Araujo, A. (2012). Análisis de los diseños de los sombreros de iraca elaborados en Colón - Génova, Nariño. Revista U.D.C.A Actualidad \& Divulgación Científica. 15(1), 227-237. https://doi.org/10.31910/rudca.v15.n1.2012.820

Fashion Revolution Colombia. (2020). El reto que enfrenta la industria colombiana de confecciones $y$ afines ante el Covid-19 [comunicado de prensa]. https://bit.ly/3kYfJyc

Fuentes, C. (octubre, 2012). Etnomatemática, geometría y cultura: el caso de los artesanos 
del municipio de Guacamayas, Boyacá. En G. Obando (Presidencia), Matemática Educativa. 13 encuentro colombiano. Conferencia llevada a cabo en el ECME 13, Medellín, Colombia.

Fuentes, C. (2010). Prácticas Cotidianas y Conocimientos Matemáticos, Estudio de Caso con Modistas en Bogotá, Colombia. Revista Latinoamericana de Etnomatemática, 3(1), 31-44.

García, J. y Bernandino, N., (2019). Conocimientos geométricos en la elaboración de un artefacto en una comunidad Ñuu Savi. Revista de Investigación Educativa de la REDIECH, 10(19), 105-120. http://dx.doi.org/10.33010/ie_rie_rediech.v10i19.634

Gerdes, P. (2013). Geometría y Cestería de los Bora en la Amazonía Peruana. Ministerio de Educación.

Gerdes, P. (2014). Reflexões sobre o ensino da matemática e diversidade cultural. Revista Latinoamericana de Etnomatemática, 7(2), 108-118.

Hernández, R. (2014). Metodología de la investigación. Industria Editorial Mexicana, Reg.

Marmolejo G. \& Vega M. (2012). La visualización en figuras Geométricas. Importancia y complejidad de su aprendizaje. Educación Matemática, 24(3), 7-32.

Mengual, E., Gorgorió, N. y Albarracín, L. (2017). Análisis de las actividades propuestas por un libro de texto: el caso de la medida. REDIMAT - Revista de Investigación en Educación Matemática, 6(2), 136-163. http://dx.doi.org/10.17583/redimat.2017.2415

Morales, M., \& Autor, A. (2019). Deconstrucción del diseño, un análisis a la base del diseño de las artesanías de Usiacurí. Revista latinoamericana de Etnomatemática, 12(2), 6-28.

Pérez, D. (2013). Una propuesta para la construcción de significado del concepto de área, en una comunidad de práctica para sexto grado. Revista Papeles, 5(9), 87-95.

Rey, M. \& Autor, A. (2011). Medición y estimación de los albañiles, un aporte a la educación matemática. Revista U.D.C.A Actualidad \& Divulgación Científica, 14(1), 137-147. https://doi.org/10.31910/rudca.v14.n1.2011.766

Romero, V., Juarez, A. \& Navarro, I. (2009). Una experiencia en Etnomatemática: Geometría del Pocho Catamarqueño. Revista de Educación Matemática, 14(1), 137-147.

Rosa, M., Lawrence, S., Gavarrete, M. y Alangui, W. (2017). Ethnomathematics and its Diverse Approaches for Mathematics Education. Springer. 
Proenza, Y. y Leyva L. (2008). Aprendizaje desarrollador en la matemática: estimulación del pensamiento geométrico en escolares primarios. Revista iberoamericana de educación, 48(1), 1-7. https://doi.org/10.35362/rie4812249

Sánchez Caro, Y. Segura Villamil, Y. (2020). Economía colombiana en tiempos de Covid-19. [Trabajo de Grado, Universidad Católica de Colombia]. https://hdl.handle.net/10983/24657

Vasilachis, I. (2006). Estrategias de Investigación Cualitativa. Gedisa Editorial.

Verner, I., Massarwe, K. y Bshouty, D. (2019). Development of competencies for teaching geometry through an ethnomathematical approach. Journal of Mathematical Behavior, 56 (100708). https://doi.org/10.1016/j.jmathb.2019.05.002

Zapata, F., \& Cano, N. (octubre, 2008). La enseñanza de la magnitud. Conferencia llevada a cabo en el Noveno Encuentro Colombiano de Matemática Educativa, Universidad Popular del Cesar, Valledupar, Cesar. 